
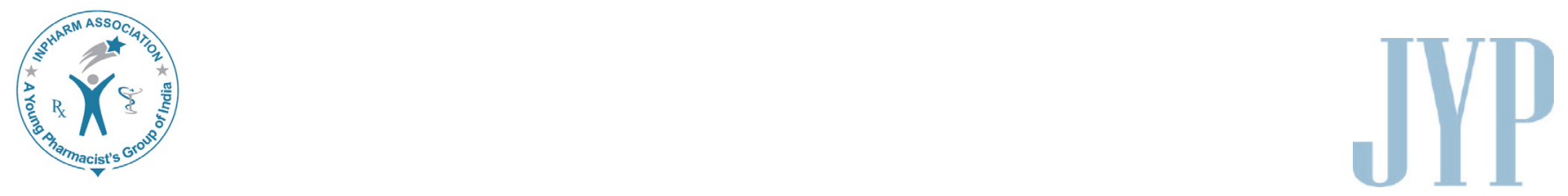

\title{
Consistency between Causality Assessments Obtained With Various Scales and Their Agreement for Adverse Drug Events Reported in Pediatric Population
}

\author{
Devang Ashwinkumar Rana, Sunilkumar Nagjibhai Bhadiyadara, Harsh \\ Jayendra Shah, Supriya Deepak Malhotra, Varsha Jitendra Patel \\ Department of Pharmacology, Smt. NHL Municipal Medical College, Ellisbridge, Ahmedabad-380006, \\ India
}

\begin{abstract}
Objective: The goal of this study was to examine correlation between various causality assessments scales and their agreement in reporting ADRs in children. Methods: All hospitalized pediatric patients were followed up for adverse drug reaction in a single pediatric unit for one year. We compared the WHO, Naranjo, CIOMS/RUCAM and French causality assessment scales in 36 identified ADRs in pediatric ward during 2012. The agreement between obtained causality assessments were analyzed by Cohen's Kappa (K) statistical test. Results: In the 290 pediatric patients, 36 adverse drug reactions were noted. Prevalence of ADR was $10.3 \%$. Maculopapular rash was most frequently observed ADR. Antimicrobials (56\%) were the most commonly involved drug group in ADR and cephalosporin's being the most frequent cause for ADR. RUCAM and French scale showed better agreement (K:0.067) with each other as compared to other scales. WHO and French scale showed least agreement (K:-0.026) with each other as compared to other scales. Naranjo's scale showed poor agreement (K:0.014) with WHO scale and worse agreement (K:-0.016) with French scale. There was no correlation between RUCAM and Naranjo's scale. Conclusion: Full agreement was not found between any of two scales of causality assessment. There was discrepancy seen between scalesdue to different definitions of causality criterias for assessing adverse drug reactions. This can influence the outcome of causality assessment significantly.
\end{abstract}

Key words: ADR, Causality scales, Children, Correlation, Kappa.

\begin{tabular}{|c|c|}
\hline \multicolumn{2}{|c|}{ Access this article online } \\
\hline Journal Sponsor & \multirow[b]{2}{*}{$\begin{array}{l}\text { Website: } \\
\text { www.jyoungpharm.org }\end{array}$} \\
\hline & \\
\hline www.phe & DOI: 10.5530/jyp.2015.2.6 \\
\hline
\end{tabular}

*Address for correspondence:

Dr. Devang A. Rana, Tutor, Department of Pharmacology, Smt. NHL Municipal Medical College, Ellisbridge, Ahmedabad-380006 India E-mail : devangandu@gmail.com

\section{INTRODUCTION}

According to $\mathrm{WHO}$ an Adverse drug reaction (ADR) is defined as "a response to a drug which is noxious \& unintended, which occurs at doses normally used in man for prophylaxis, diagnosis or therapy of disease or for modification of physiological function excluding failure to accomplish the intended purpose". ${ }^{1}$ 


\begin{tabular}{lcccc}
\multicolumn{4}{l}{ Table 1: Age and gender distribution of ADR } \\
Age (years) & Male & Female & Total & Percentage \\
$\mathbf{0 - 1}$ & 4 & 1 & 5 & 16.66 \\
$\mathbf{1 - 3}$ & 3 & 1 & 4 & 13.33 \\
$\mathbf{3 - 5}$ & 7 & 3 & 10 & 33.33 \\
$\mathbf{5 - 1 0}$ & 2 & 4 & 6 & 20 \\
$\mathbf{1 0 - 1 2}$ & 2 & 3 & 5 & 16.66 \\
Total & 18 & 12 & 30 & 100
\end{tabular}

ADRs are a cause of significant indisposition \& transience in patients. They are responsible for increased number of hospital admissions. It has been estimated that majority of ADRs are preventable. ${ }^{2}$ The incidence and severity of ADRs can be influenced by age, gender, concurrent renal , hepatic disease, genetic factors, type of drug, route of administration, duration of therapy, and dosage. ${ }^{3,4}$

Paediatric patients constitute a vulnerable group with regard to rational drug prescribing. Paediatricians often prescribe drugs in an 'off-label' manner to children, thereby increasing the risk of drug toxicity. Adequate controlled clinical trials in children are lacking, mainly because of issues of cost and responsibility, and to regulations that frequently act as major obstacles. Most of the ADRs, observed in paediatric studies, mainly effect the skin (rash, urticaria) and the gastrointestinal system (diarrhoea, nausea and vomiting), but we can also observe involvement of other systems. The drugs most frequently associated with adverse reactions are antibiotics, antipyretics and non -steroidal anti-inflammatory. ${ }^{5-7}$

Hence, the present study was undertaken to evaluate the clinical spectrum of all paediatric ADRs in patients admitted to a paediatric unit of tertiary care teaching hospital. It also emphasises on the need and importance of an effective pharmacovigilance programme.

\section{MATERIALS AND METHODS}

The study was carried out in a paediatric unit at a tertiary care teaching hospital. The study was a continuous, observational, longitudinal, prospective, non-interventional carried out from December 2010 to December 2011. Protocol of the study procedure is formed first together with the Case Record Form (CRF), Patient Information Sheet and Informed Consent Form. The protocol submitted to the institutional ethics committee (IEC) for approval. Written consent was obtained from patient's parents/ guardian before enrolment after explaining the aim of the study. After taking written consent, the data was collected in CDSCO (Central Drugs Standard Control Organization) adverse drug reaction (ADR) reporting form. ADR reporting form includes patient's demographic details, description of suspected adverse event, details of suspected medication, information about reporter and causality assessment details. All children admitted in a paediatric unit of Sheth V.S. Hospital who are prescribed any drug observed prospectively for any adverse event. Those who develop adverse event followed up \& along with recording of their cases till they discharged. Monitoring for adverse effects was based on regular questioning of caretaker and medical professionals for occurrences of ADRs. Laboratory investigations were done if indicated clinically and for confirmation of ADRs. All the records then analyzed for calculating the prevalence of ADR \& also to find out pattern and causality of drug reactions. All the information was carefully recorded in CDSCO Suspected ADR reporting form and analysed for type of ADR, ${ }^{8}$ assessment of severity per Modified Hartwig and Siegel Scale, ${ }^{9,10}$ its seriousness, to determine preventability using Criteria of Schumock and Thornton. ${ }^{11,12}$ Causality Assessment using WHO Criteria, Naranjo's scale, ${ }^{13}$ Benichou system (RUCAM- Roussel Uclaf Causality Assessment Method), ${ }^{14}$ French Imputation system.

\section{STATISTICAL ANALYSES}

All the data was compiled in excel sheet and subjected to descriptive statistical analysis. Causality assessment by different criteria's was compared for agreement using Cohen's kappa test.

\section{RESULT}

A total of 290 paediatric patients from a single unit were enrolled in study. Out of these, 30 patients were having suspected adverse drug reactions. Among these 30 cases, 36 adverse drug reactions were notified hence prevalence of ADR was $10.34 \%$.

\section{Age and gender distribution of ADR}

Out of 30 patients, 18 (60\%) were male and $12(40 \%)$ were female. Maximum patients belonged to the age group of $3-5$ year $(33.33 \%)$ followed by $5-10$ year (20\%) (Table 1 )

\section{Type of ADRs}

Total of 36 ADRs were observed in 30 patients during one year of study in single unit. The most common variety of drug reactions were maculopapular rashes $(16.7 \%)$ followed by diarrhea $(13.9 \%)$ and abdominal pain $(11.1 \%)$. Most commonly affected system was skin (41.7\%) followed by 
gastrointestinal system $(33.3 \%)$.

\section{Type of adverse drug reaction}

The observed adverse drug reactions were classified into two principal types as per Rawlins \& Thompson's classification 14 cases were augmented type (36.1\%) and 22 cases were bizarre type (63.9\%)

\section{Responsible Drugs}

Table 2. Shows the major drug group's involved and detailed list of various drugs that were implicated in adverse drug reaction during study period.

\section{Duration between introduction of drug and onset of reaction (Incubation period)}

\section{Causality assessment using WHO - UMC criteria}

Shows causality assessment of adverse reactions according to WHO-UMC criteria. 35 cases were of probable type $(97.2 \%) \& 1$ case was of unclassified type $(2.8 \%)$

\section{Causality assessment using NARANJO's criteria}

Shows causality assessment according to Naranjo's criteria. It's a questionnaire based scoring system. 12 cases were of probable category (score+6) $(33.3 \%), 3$ cases were of possible category $($ score +4$)(8.3 \%) \& 21$ cases were of possible category (score+3) (58.3\%).

\section{Comparison between different causality assessments scales} using Cohen's Kappa test (Table 3)

Comparison of WHO and Naranjo's causality shows number of agreements 12 (33.33\% of observations) (kappa $=0.014)$ hence strength of agreement is "poor".

Comparison of WHO and RUCAM causality shows number of agreements 29 (80.56\% of observations) (kappa $=-0.024)$ hence strength of agreement is worse than what we expect to see by chance alone.

Comparison of WHO and French causality shows number of agreements 22 (61.11\% of observations) (kappa $=$ -0.026) hence strength of agreement is worse than what we expect to see by chance alone.

Comparison of Naranjo's and French causality shows number of agreements 15 (41.67\% of observations) (kappa $=-0.016)$ hence strength of agreement is worse than what we expect to see by chance alone.

Comparison of RUCAM and French causality shows number of agreements 6 (16.67\% of observations) (kappa $=0.067)$ hence strength of agreement is "poor".

$\begin{array}{lccc}\text { Table 2: Responsible drugs } & \text { Frequency of } \\ \text { Drug Group } & \text { Drug } & 23 & \text { Percentage } \\ & - & 2 & 56.09 \\ & \text { Aminoglycosides } & 4 & 4.87 \\ \text { Antimicrobials } & \text { Penicillins } & 2 & 9.75 \\ & \text { Tetracyclines } & 10 & 4.87 \\ & \text { Cephalosporins } & 2 & 24.39 \\ & \text { Macrolides } & 3 & 4.87 \\ & \text { Cotrimoxazole } & 10 & 7.13 \\ \text { Antimalarial } & - & 6 & 24.39 \\ & \text { Artesunate } & 3 & 14.63 \\ \text { Antiepileptics } & \text { Chloroquine } & 1 & 7.31 \\ & \text { Primaquine } & 3 & 2.43 \\ & - & 3 & 7.13 \\ \text { Others } & \text { Phenytoin } & 5 & 7.13 \\ & - & 2 & 12.19 \\ & \text { Enalapril } & 1 & 4.87 \\ & \text { ORS } & 2 & 2.43 \\ & \text { Diazepam } & 4.87\end{array}$

\begin{tabular}{lcccc}
\multicolumn{4}{l}{ Table 3: comparison between different causality scales } & \\
& WHO CAUSALITY & NARANJO'S SCORE & RUCAM CAUSALITY & French \\
Who causality & & 0.014 & -0.024 & -0.026 \\
Naranjo's score & 0.014 & & 0.000 & -0.016 \\
Rucam Causality & -0.024 & 0.000 & & 0.067 \\
French & -0.026 & -0.016 & 0.067 &
\end{tabular}




\section{Assessment of Severity using modified Hartwig and SiegeI scale.}

32 cases were of moderate type (88.9\%), 3 cases were of severe type $(8.3 \%) \& 1$ case was of mild type $(2.8 \%)$

\section{Assessment of Seriousness}

4(11.11\%) children were hospitalised and were considered serious as per WHO definition of serious adverse drug reaction

\section{Assessment of Preventability}

Preventability of adverse drug reaction was assessed by Schumock \& Thornton criteria. ${ }^{11,12}$ Most of the cases were probably preventable $(97.22 \%)$. Almost all cases (35) were "probably preventable". Only one case of ORS induced hypernatremia was "definitely preventable" as dose and frequency of administration were inappropriate for patient's age, weight and disease state.

\section{Outcome of ADRs}

All 36 cases of adverse drug reactions recovered completely without any sequelae.

\section{DISCUSSION}

Children are considered as "Therapeutic orphan" worldwide. Hence they are at increased risk of therapeutic failure and ADRs continue to cause unnecessary disability and death among them.15 Despite efforts being made to reduce the incidence of medication related adverse events, the morbidity, and mortality especially in paediatric population due to drug-induced reactions continue to be unacceptably high.16 Keeping these objectives in mind, a prospective hospital based observational study was carried out for duration of one year in children admitted to a paediatric unit of tertiary care teaching hospital. It also emphasises on the need and importance of an effective pharmacovigilance programme. During this period, a total of 36 adverse drug reactions in 30 children were recorded $\&$ analyzed further

\section{Age and gender distribution of ADR}

Majority of patients in our study who had adverse drug reactions were males $(60 \%)$ as compares to females $(40 \%)$. Children of 3-5 year age group (33.33\%) were more susceptible to ADRs among paediatric patients. (Table1). However, in earlier study it was found that $63 \%$ were females and 37\% were males. Infants less than 1 year of age $(60 \%)$ were more susceptible for ADRs, the difference in various studies may be related to the regional variations in health care seeking behavior of population. ${ }^{17}$

\section{Types of ADR}

Of the various types of adverse drug reactions seen in our study maculopapular rash $(16.7 \%)$ followed by diarrhoa (13.9\%) and abdominal pain (11.1\%). (Table 2) In previous studies the most common ADRs were rash, urticaria followed by fever,anaphlactic shock,vomiting,chills and rigors. ${ }^{5}$ A study from North India ${ }^{17}$ also found maculopapular rash to be most common type of ADR. In a study conducted in Nigeria in childern, two most commonly reported ADRs were diarrhoea (51\%) and skin rashes $(18 \%)$ which is in concordance with our study. ${ }^{18}$ This variation could be due to difference in the drug usage and difference in ethnic group in different parts of our country.

\section{Type of Adverse Drug Reaction}

Majority of adverse drug reactions 22 (63.9\%) were of Type $B$, since these reactions were totally bizarre effects that are not to be expected from the known pharmacological actions of a drug, when given in the usual therapeutic doses to a patient, whose body handles the drug in the normal way. The remaining 14 (38.8\%) ADRs belonged to Type $\mathrm{A}$, since these reactions were the result of an exaggerated, but otherwise normal, pharmacological action, of a drug given in usual therapeutic doses. (Table 4$)^{19}$ recorded $96 \%$ Type $\mathrm{B}$ reactions and only $4 \%$ Type $\mathrm{A}$ in their study which is in concordance with our study.

\section{Responsible Drugs}

Most frequently reported adverse drug reactions were for Antimicrobial agents in 23 cases (56.09\%) followed by antimalarials - 10 cases $(24.39 \%)$ and Antiepileptics -3 cases $(7.13 \%)$. The antibiotics associated with ADRs include cephalosporins, penicillins, cotrimoxazole, aminoglycosides, tetracyclines and macrolides in order of significance. Among antimalarials, artesunate was most commonly associated with occurrence of ADRs. Other studies also reported antibiotics as the major group causing

\begin{tabular}{lcc}
\hline $\begin{array}{l}\text { Table 4: Management of ADR } \\
\text { Management }\end{array}$ & No. of Cases & Percentage \\
\hline $\begin{array}{l}\text { No action taken } \\
\text { Suspected drug } \\
\text { discontinued }\end{array}$ & 9 & 25 \\
$\begin{array}{l}\text { \& symptomatic } \\
\text { treatment }\end{array}$ & 19 & 52.8 \\
$\begin{array}{l}\text { Suspected drug } \\
\text { discontinued but } \\
\text { no other action } \\
\text { taken }\end{array}$ & 6 & \\
$\begin{array}{l}\text { Dose of suspected } \\
\text { drug modified }\end{array}$ & & 16.7 \\
Total & 2 & \\
\hline
\end{tabular}

Journal of Young Pharmacists Vol 7 • Issue 2 • Apr-Jun 2015 


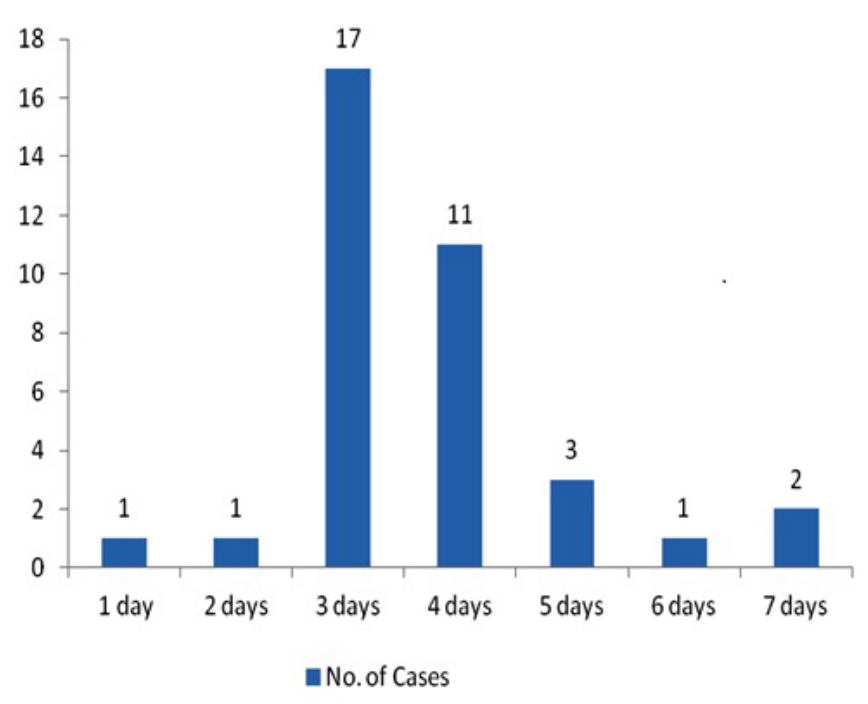

Figure 1: Duration of ADR

adverse drug reactions followed by antiepileptics and general anaesthetics. ${ }^{5}$ Reason for this was they had included paediatric surgery department and neonatal intensive care unit which was not included in our study and we focused on patients admitted in single paediatric unit.

\section{Duration between introduction of drug and onset of reaction (Incubation period)}

Majority of the reactions $(77.77 \%)$ were occurred within 3-4 days of taking the suspected drug. However, cases as early as 2 hours and even after 7 days were also reported. (Figure 1)

\section{Causality Assessment}

There is no gold standard investigation for confirmation of a drug-induced reaction. Instead diagnosis and assessment of a drug cause involve analysis of a constellation of features such as timing of drug exposure and reaction time, course of reaction with drug withdrawal/discontinuation, timing and nature of a recurrent eruption on rechallenge, a history of similar reaction to the suspected drug and previous reports of similar reactions to the same drug. ${ }^{20}$ In this study, WHO and Naranjo's causality criterias were used to categorize the ADRs into definite, probable and possible categories as they are very simple and widely accepted method to assess causality.

In our study, 36 cases of ADR were seen. Dechallenge was done in 25 cases, dose reduced in 3 cases and doses not changed in 9 cases. According to WHO causality definition, 35 cases $(97.2 \%)$ were "probable". The remaining one case $(2.8 \%)$ was "unclassifiable". İn this case, ADR was erythmatous rashes most likely due to two antimicrobials- cefoperazone sulbactum and piperacillin tazobactum along with severeal other antimicrobials. In a previous study, causality was classified as 'definite' (44.1\%), 'probable' $(49.9 \%)$ or 'possible' $(6.0 \%)$ according to WHO criteria. ${ }^{21}$ In our cases, rechallenge was not done because of alertness of the treating clinicians.

In our study, according to Naranjo's causality questionnaire, 24 cases $(66.6 \%)$ were "probable" and 12 cases $(33.3 \%)$ were of "possible" category..In an Indian study by Priyadharsini R et al. (2011), 80\% ADRs were of "probable", 17\% were of "possible" and 3\% were definite causality according to Naranjo's criteria. No ADR of definite category was observed in our study as rechallenge was not done.

In our study, two other causality scales were used : Benichau causality system and French imputation system for assessment. According to Benichau (RUCAM) causality system, 30 cases $(83.2 \%)$ were probable and 6 cases $(16.6 \%)$ were possible. According to French imputation system, 23 cases (63.88\%) were possible (I2) and 13 cases (36.11\%) were likely (I3) type. Hence probably French system provides wider sprectum of causality compared to other three methods.

Comparison of strength of agreement between different scales of causality assessment by using Cohen's kappa test. İt shows that full agreement was not found between any of two scales of causality assessment. Positive but poor agreement based on kappa values was seen between WHO and Naranjo's causality comparison and between RUCAM and French causality comparison. Negative agreement based on kappa values was seen between WHO and RUCAM, WHO and French and Naranjo's and French causality comparison. There was discrepancy seen between scales. This was due to different definitions of causality criterias for assessing adverse drug reactions. The differences in our study and studies may be due to different scales used for causality assessment or because of individual differences in the interpretation of data.

\section{Assessment of severity}

Modified Hartwig and Siegel scale ${ }^{9,10}$ was used for assessment of severity. 32 adverse drug reactions $(88.9 \%)$ were of moderate severity as they didn't require any specific therapy. They were simply managed by withdrawal of the suspected drug \& supportive treatment. 3 adverse drug reactions $(8.3 \%)$ were severe and required immediate cessation of the suspected drug, hospitalization \& intensive medical care. The results comply with earlier study in which $77 \%$ had moderate and $23 \%$ had severe adverse 
drug reactions. ${ }^{5}$

\section{Assessment of seriousness}

Adverse drug reactions were considered serious on the basis of WHO definition of serious adverse reaction ${ }^{22}$ During our study, 4 serious reactions were reported one patients was of hypernatremia, one was of thrombocytopenia, one was of erythema multiform and one was of blurring of vision. All these reactions were considered to be serious as they required prolongation of hospital stay and intensive medical care.

\section{Assessment of preventability}

Schumock \& Thornton criteria ${ }^{11}$ was used for assessment of preventability. 35 reactions $(97.22 \%)$ were considered probably preventable as they involved poor patient compliance, potential drug interaction and failure to carry out therapeutic drug monitoring. The remaining one reaction $(2.77 \%)$ was regarded as definitely preventable. The results comply with earlier study in which $87 \%$ had probable, $3 \%$ had definitely and $10 \%$ had no preventability. ${ }^{5}$

\section{Management of ADR}

$52.8 \%$ ADRs $19 / 36$ were managed just by cessation of the suspected drug and supportive or symptomatic treatment. 6 ADRs (16.7\%) required immediate cessation of suspected drug, hospitalization of the patient \& intensive medical treatment. 5.6\% ADRs (2/36) required modification of the dose as these were dose related adverse effects. In 9 cases, no action had been taken.

ADRs rank as one of the top ten leading causes of illness and death in the developed world. Children are known to be at greater risk than adults, there is a remarkable lack of understanding of causation and therefore the ability to avoid or prevent these occurrences is limited. The safety of drugs and ADR profile is less monitored in children Hence a prospective hospital based observational study was carried out for duration of one year to monitor ADRs occurring in children admitted to a paediatric unit of tertiary care teaching hospital. It also emphasised on the need and importance of an effective pharmacovigilance programme. A wide clinical spectrum of ADRs ranging from mild abdominal pain to serious hypernatremia, thrombocytopenia was observed.

Prevalence of ADR was found to be $10.34 \%$. Majority of children with ADRs were belonged to age group of 3-5 years among paediatric patients. There was slight male preponderance seen with M:F ratio being 3:2. Most frequent adverse drug reactions reported were maculopapular rash followed by diarrhoa and abdominal pain in decreasing order of frequency. Majority of adverse drug reactions (63.9\%) were Bizarre/Unpredictable (Type B) and occurred due to hypersensitivity reaction. The remaining (38.8\%) ADRs were belonged to Augmented/predictable (Type A).

Majority of the reactions $(77.77 \%$ ) occurred within 3-4 days of taking the suspected drug. However, cases as early as within 2 hours and even after 7 days were also reported. Using WHO-UMC causality definitions, $97.2 \%$ of ADRs were "probable" and only $2.8 \%$ were of "unclassifiable" in nature.Using Naranjo's causality questionnaire, 66.6\% of ADRs were "probable" and 33.3\% were of "possible" category. According to Benichou (RUCAM) causality system, $83.2 \%$ were probable and $16.6 \%$ were possible. According to French imputation system, 63.88\% were possible and $36.11 \%$ were likely type. Using Cohen's kappa test. Positive but poor agreement based on kappa values was seen between WHO and Naranjo's causality comparison and between RUCAM and French causality comparison.

\section{CONCLUSION}

Antimicrobials were most frequently associated with ADRs followed by antimalarials. Almost all ADRs reported in this study were probably preventable. Drugs should be used only if specifically indicate and polypharmacy should be avoided. Careful drug history should be taken beforehand to exclude drug allergies and document all drugs already in use (including over the counter products). Drug therapy should be individualized based on age, weight, body surface area, disease states etc. Proper Instruction should be given to patients and guardians carefully on nature of drug and proper mode of use. Health professionals and pharmacologists ${ }^{23}$ should periodically educate about ADRs and technical aspects of drug monitoring process through pharmacovigilance programme. The awareness of spontaneous reporting of ADRs among health care professionals and general population should be given due considerations for preventing the morbidity and mortality among the paediatric population. Train health professionals in drug safety and cultivate habit of rational drug use in them from the beginning to manage up to serious ADR.

\section{CONFLICTS OF INTEREST}

Authors declared no conflict of interest. 


\section{ACKNOWLEDGEMENT}

We are thankful to department of pediatrics and to the dean of Smt. NHL medical College, Dr. Pankaj R. Patel for permitting the study to take place in institute.

\section{REFERENCES}

1. WHO, International drug monitoring. Role of national centres. WHO Technical report series no. 498. Geneva, Switzerland; 1972.

2. Hakkarainen KM, Hedna K, Petzold M, Hägg S. Percentage of Patients with Preventable Adverse Drug Reactions and Preventability of Adverse Drug Reactions - A Meta-Analysis. PLoS ONE. 2012; 7(3): e33236.

3. Muaed Jamal Alomar. Factors affecting the development of adverse drug reactions. Saudi Pharm J. Apr 2014; 22(2): 83-94.

4. Edwards IR. In: Speight, T.M. and Nicholas, H.G., Eds., Avery's Drug Treatment, 4 Ed. Adis International; 1997. 261.

5. Priyadharsini R, Adithan C, Sreenivasan S. A study of adverse drug reactions on pediatric patients. Journal of pharmacology \& pharmacotherapeutics 2011; 2(4): 277-80.

6. Children and ADR, Napoleone Italian Journal of Pediatrics 2010 , 36:4 Available online at http://www.ijponline.net/content/36/1/4. [Last accessed on 05/12/2014.]

7. Le J, Nguyen T, Law AV, Hodding J. Adverse drug reactions among children over a 10-year period. Pediatrics 2006; 118(2): 555-62.

8. Laurance DR, Bennett PN, Brown MJ. Clinical pharmacology. 8th edition. Singapore: Churchill livingstone; 1997. 121-37.

9. Adverse drug reactions In: The Merck manual of diagnosis \& therapy. 1999. Available from: http://www.merck.com/sec-2/ chap302.[Last accessed on 05/12/2014.]

10. Hendrick AE, McCarthy MW, Hofer K. University of Virginia Health System Adverse Drug Reaction Reporting Program Policy and Procedure. University of Virginia Health System. Department of Pharmacy Services, Drug Information Center.1-5-99. http://hsc. virginia.edu/pharmacy-services/ADRRP/ADRP\& P.html.

11. Lau PM, Stewart K, Dooley M. The ten most common adverse drug reactions in oncology patients: do they matter to you? Support care cancer 2004; 12(9): 626-33.

12. Schumock $G$, Thornton J. Focusing on the preventability of adverse drug reactions. Hosp Pharm. 1992; 27(6): 538.
13. Naranjo CA, Busto U, Sellers EM, Sandor P, Ruiz I, Roberts EA, et al. A method for estimating the probability of adverse drug reactions. Clin Pharmacol Ther. 1981; 30(2): 239-45.

14. Christian benichou. Adverse drug reaction: a guide to diagnosis and management; 1994. 277-82.

15. Bruce Carleton, Anne Smith. Canadian Paediatric Surveillance ProgramRecognizing and Preventing Adverse Drug Reactions in Children. Pharmaceutical Outcomes Programme, Children's \& Women's Health Centre of British Columbia. 2010 Available online http://www.cpsp.cps.ca/uploads/publications/Results-2010.pdf. [last accessed on 5/12/2014]

16. Berry MA, Shah PS, Brouillette RT, Hellmann J. Predictors of mortality and length of stay for neonates admitted to children's hospital neonatal intensive care units. J Perinatol. 2008; 28(4): 297-302.

17. Sharma VK, Sethuraman G, Kumar B. Cutaneous adverse drug reactions: Clinical pattern and causative agents-A six-year series from Chandigarh, India. J Postgrad Med. 2001; 47(2): 95-9.

18. Oshikoya KA, Senbanjo IO, Njokanma OF. Parental reporting of suspected adverse drug reactions in children in Lagos. Nigeria. Arch Dis Child. 2009; 94(6): 469-73.

19. Ghosh S, Afcharya LD, Rao PGM. Study and evaluation of the various cutaneous adverse drug reactions in Kasturba hospital, Manipal. Indian J Pharm Sci. 2006; 68(2): 212-5.

20. Shear NH, Knowles SR, Sullivan JR, Shapiro L. Cutaneous reactions to drugs. In: Freedberg IM, Eisen AZ, Wolff K, editors. Fitzpatrick's dermatology in general medicine. 6th Ed. USA: McGraw Hill, Medical publishing division; 2003. p. 1330-6.

21. Mary Temple E, Renee Robinson F, Juile Miller. Frequency and preventability of adverse drug reaction in paediatric patients. Drug safety $2004 ; 27(11): 819-29$.

22. National pharmacovigilance protocol. Ministry of health \& family welfare, Govt of India. 2013. Available from www.jipmer.edu/charu/ NPVP\%20for\%20Web.doc. [Last accessed on 05/12/2014]

23. Singh $H$, Dulhani N, Kumar BN, Singh P, Tewari P, Nayak K. A Pharmacovigilance Study in Medicine Department of Tertiary Care Hospital in Chhattisgarh (Jagdalpur), India. Journal of Young Pharmacists 2010; 2(1): 95-100. 